\title{
REVSED BoneJ2 - refactoring established research software
}

\section{[version 2; peer review: 3 approved]}

\author{
Richard Domander ${ }^{1}$, Alessandro A Felder ${ }^{1,2}$, Michael Doube (iD) 1,3 \\ ${ }^{1}$ Department of Comparative Biomedical Sciences, The Royal Veterinary College, University of London, London, NW1 0TU, UK \\ ${ }^{2}$ Research Software Development Group, University College London, London, WC1E 7HB, UK \\ ${ }^{3}$ Department of Infectious Diseases and Public Health, City University of Hong Kong, Kowloon, Hong Kong
}

V2 First published: 22 Feb 2021, 6:37

https://doi.org/10.12688/wellcomeopenres.16619.1

Latest published: 28 Apr 2021, 6:37

https://doi.org/10.12688/wellcomeopenres.16619.2

\section{Abstract}

Research software is often developed with expedience as a core development objective because experimental results, but not the software, are specified and resourced as a project output. While such code can help find answers to specific research questions, it may lack longevity and flexibility to make it reusable. We reimplemented BoneJ, our software for skeletal biology image analysis, to address design limitations that put it at risk of becoming unusable. We improved the quality of BoneJ code by following contemporary best programming practices. These include separation of concerns, dependency management, thorough testing, continuous integration and deployment, source code management, code reviews, issue and task ticketing, and user and developer documentation. The resulting Bone 2 represents a generational shift in development technology and integrates with the ImageJ2 plugin ecosystem.

\section{Keywords}

Open-source, Java, Bone, Biology, Morphometry, ImageJ, FIJI, BoneJ, Image analysis, Software engineering, Programming

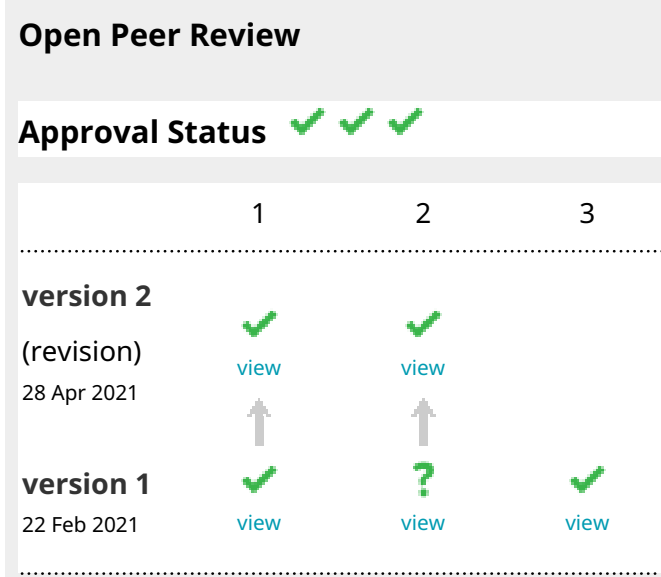

1. Loic Courtois, 3Dmagination Ltd, Didcot, UK Kamel Madi, 3Dmagination Ltd, Didcot, UK

2. Mariana Kersh ID, University of Illinois at Urbana-Champaign, Urbana, USA University of Illinois at Urbana-Champaign, Urbana, USA

3. Deborah Schmidt iD, Max Delbrück Center for Molecular Medicine in the Helmholtz Association, Bonn, Germany

Any reports and responses or comments on the article can be found at the end of the article. 
Corresponding author: Michael Doube (mdoube@cityu.edu.hk)

Author roles: Domander R: Conceptualization, Data Curation, Formal Analysis, Investigation, Methodology, Project Administration, Software, Validation, Visualization, Writing - Original Draft Preparation, Writing - Review \& Editing; Felder AA: Conceptualization, Data Curation, Formal Analysis, Investigation, Methodology, Project Administration, Software, Validation, Visualization, Writing - Original Draft Preparation, Writing - Review \& Editing; Doube M: Conceptualization, Data Curation, Formal Analysis, Funding Acquisition, Investigation, Methodology, Project Administration, Resources, Software, Supervision, Validation, Visualization, Writing - Original Draft Preparation, Writing - Review \& Editing

Competing interests: No competing interests were disclosed.

Grant information: RD was supported by a Wellcome Trust Biomedical Resource and Technology Development Grant (108442/Z/15/Z), and AAF by a UK Biotechnology and Biological Sciences Research Council Project Grant (BB/P006167/1) and an RVC PhD studentship (University of London, Thomas Brown Animal Fund). Collaboration with Kyle Harrington (University of Idaho) on SciView was supported by a Royal Society International Exchanges Scheme grant (IES/R3/170078). Early work on BoneJ2 was the subject of a Software Sustainability Institute Open Call for Collaborations.

The funders had no role in study design, data collection and analysis, decision to publish, or preparation of the manuscript.

Copyright: (c) 2021 Domander R et al. This is an open access article distributed under the terms of the Creative Commons Attribution License, which permits unrestricted use, distribution, and reproduction in any medium, provided the original work is properly cited.

How to cite this article: Domander R, Felder AA and Doube M. Bone]2 - refactoring established research software [version 2; peer review: 3 approved] Wellcome Open Research 2021, 6:37 https://doi.org/10.12688/wellcomeopenres.16619.2

First published: 22 Feb 2021, 6:37 https://doi.org/10.12688/wellcomeopenres.16619.1 


\section{REVISED Amendments from Version 1}

Fixes a typo and updates details to match new GUI elements in step 11 of the interactive use case.

Adjusts table of expected values to match those from following precisely the steps in the Interactive Use Case. There is a small difference in automatic threshold if the user clicks 'Auto' prior to clicking 'Apply' in the Threshold window, which results in a slight difference in BV and connectivity. This discrepancy is also present if the macro command setAutoThreshold is used in the scripting example, so it has been replaced with setThreshold $(26892,65535)$, which ensures the same threshold values are used in the scripting and interactive cases. Note that this is a good example of how even minor variations in image processing parameters can affect readout values.

Updates the user documentation URL to https://imagej.github.io/plugins/bonej

Updates the DOI (10.5281/zenodo.4635373) and version (styloid-r11) to the current ones.

Fixes a number of small typos in the text.

Any further responses from the reviewers can be found at the end of the article

\section{Introduction}

Bone $\mathrm{J}^{1}$ is a collection of bone image analysis plugins for the Image $\mathrm{J}^{2}$ scientific image analysis software. A plugin is a piece of software that extends the capabilities of its parent application. For example, BoneJ provides a tool that counts connections within a structure by calculating an image's Euler characteristic $(\chi)$, a functionality that is absent from core ImageJ. Both ImageJ and BoneJ are free and open-source, which means that their source code is readily available online for anyone to inspect, download, use, modify and distribute, taking an open science approach by providing all methodological details. ImageJ plugins run on Windows, MacOS, GNU/Linux, and other operating systems because they are implemented in Java. Java programs are executed inside an operating system-agnostic Java Runtime Environment (JRE).

The plug-ins in BoneJ are intended for analysing computed tomographic (CT) and X-ray microtomography (XMT) images of whole bones, trabecular bone, and osteocyte lacunae. BoneJ was originally produced to satisfy a need to answer research questions using data to which extant software was ill-suited ${ }^{3-5}$. To enable other researchers to benefit from the development effort, plugins written by Doube and others were altered to suit the skeletal research domain (for example by using ASBMR standard histomorphometric nomenclature ${ }^{6}$ ), bundled together in a single Java archive file (BoneJ.jar) for ease of installation, and basic user and developer documentation provided at bonej.org. BoneJ became popular for bone morphometry, and in other fields such as materials, soil, and food science. By 2020 its paper $^{1}$ was being cited in peer-reviewed literature 4-5 times per week .

In the time since BoneJ was released in 2010, the software ecosystem on which it relied made several advances. Schindelin and others implemented a software updater for ImageJ as part of their Fiji Is Just ImageJ (Fiji) project ${ }^{7}$, while ImageJ2 introduced a new $N$-dimensional image model and modern development practices such as modularity, dependency management (Maven), continuous integration (Jenkins, then Travis, likely to be superseded by GitHub Actions), and community development and support protocols (GitHub, Gitter, image.sc forum) ${ }^{8}$. At the same time the JRE version 6 that BoneJ was built for was phased out, the 3D libraries BoneJ's plugins relied on (Java3D 1.5) were obsoleted, and the 3D Viewer ${ }^{9}$ that BoneJ used for surface meshing and visualisation was abandoned. BoneJ was dependent on third-party software that was no longer maintained, while users found they could not install and run BoneJ in their environment creating a risk of forced obsolescence.

At the same time, the developments in ImageJ2 and Fiji represented an opportunity to improve BoneJ's engineering standard and user experience, as well as opening avenues for new algorithm development and implementation. ImageJ2's new Ops framework and dependency management with Maven meant that libraries and plugins that had been copy-pasted into BoneJ_.jar, such as Analyze Skeleton ${ }^{10}$, could be removed and used from a single location rather than duplicated, eliminating the overhead involved in maintaining copies of active projects. Maven combined with the ImageJ updater enabled updating resources such as the linear algebra library JAMA (last updated in 2012) to JOML (under active development) and to use Eclipse Collections' fast IntHashSet implementation in place of the standard Java HashSet<Integer $>$.

'https://scholar.google.com/scholar?cites=16814410598979768336\&scisbd=1 
Due to the increased complexity and engineering sophistication of the new ImageJ 2 ecosystem, it became infeasible for BoneJ to remain a part-time, scientist-maintained project. This was especially so because as university faculty MD no longer had the long stretches of time needed to concentrate on software engineering, nor were software outputs or community support part of his employment performance criteria. In the UK, an awareness of Research Software Engineers (RSE) as valuable team members was growing ${ }^{11}$. Here, we describe the steps taken to modernise the BoneJ project, and use BoneJ to provide a perspective on the role software engineering takes in the contemporary research ecosystem.

\section{Design}

The top-level project identity has remained at bonej.org, which serves to direct users to project functions on third-party sites, rather than hosting content. The only content that remains in place at bonej.org is legacy documentation, for reproducibility purposes. Links at bonej.org direct users to user documentation at imagej.github.io, to the user forum at forum.image.sc, to developer resources including code and technical documentation at GitHub, and BoneJ's social media account on Twitter. Project Java package declarations are now all in the form of org.bonej.package, to emphasise common ownership as distinct from the personal ownership implied by org.doube.bonej.package used in BoneJ1's package declarations. Anonymous opt-in usage data are collated at Google Analytics, which helps determine which plugins are used most heavily, and which BoneJ, Java, and operating system versions are active in the user community.

The starting point of designing BoneJ2's codebase and development environment was to assess how the plugins in BoneJ1 needed to change to follow software development best practices, and to work with the new Image J platform. At the time (2016) BoneJ was one of the first projects attempting to transition to the new technology underlying Image 2 . As pioneers we wanted not only to improve our own code, but also to provide an example that others could follow. After initial research into the design of BoneJ2, we devised a strategy for restructuring the software using wrapper plugins and proceeded to work on individual tools on a case-by-case basis.

The wrapper plugins in BoneJ2 are intended to be light pieces of software that handle user interaction and call code from elsewhere. They orchestrate the multiple phases of execution that are needed to derive the results that users need to analyse their images (Figure 1). Wrapper plugins also contain code specific to skeletal biology

\section{BoneJ}

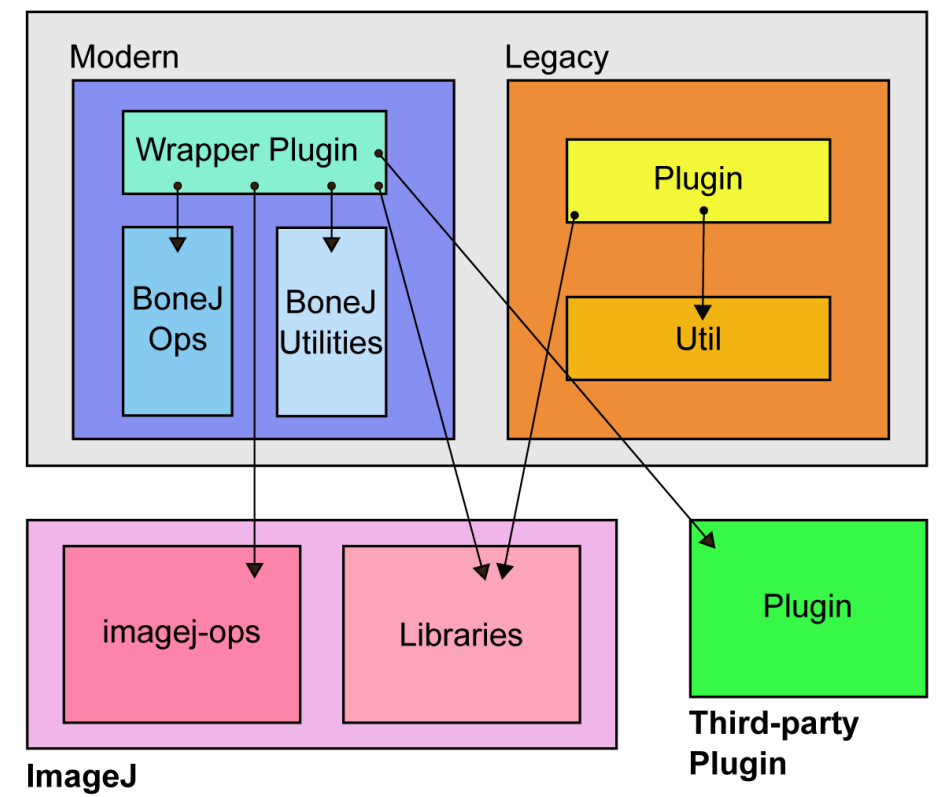

Figure 1. An overview of BoneJ architecture. Modern wrapper plugins call both ImageJ and third-party plugin code. They mainly run algorithms from the Imagej Ops framework. Both Modern and Legacy plugins depend on various Imagej libraries. Image Ops and library architecture are described in more detail elsewhere ${ }^{8}$. The presence of all the components necessary to run BoneJ is managed by Maven on the developer side and by the ImageJ Updater on the user side. 
such as bone morphometry acronyms. Code common to all the wrappers is in a base class, BoneJCommand, which wrapper plugins access via Java inheritance to avoid code duplication. Using wrappers offers advantages such as reducing the amount of code to maintain and allowing rapid implementation of changes if a certain piece of software no longer serves our needs.

Wrapper plugins were designed to call code from external sources such as the ImageJ Ops framework and third party plugins. However, for some of the wrappers we needed to provide these "external" tools ourselves. We saw that we had to rewrite the majority of BoneJ1, and that some of the functionality there was generic and useful enough to become parts of ImageJ itself. We split the previously monolithic design into independent, reusable pieces, and made BoneJ2 a much lighter distribution. We wanted to further a design where the slight differences in functionality that different audiences need, such as domain-specific terms for measurements, could be added on top of a more basic, shared technology, enabling custom "remixes" of generic image analysis algorithms.

Ideally BoneJ2 would have only modern external dependencies. However, some necessary third-party tools had not been ported to the new technology, and it would be an unreasonable workload for us to reimplement them. Some third-party code will never be modernised because it is not maintained, or if it is maintained the maintainers may not want to modernise it in the near future. As a compromise we designed modern wrappers that encapsulate calls to legacy dependencies. Mixing modern and legacy code in ImageJ can introduce unwanted complexity so we limited them to as few carefully crafted code snippets as possible.

In the early stages of BoneJ2's project development it became apparent that it would take significantly longer to port BoneJ1 than initially anticipated. We intended to release BoneJ2 only once porting was complete but realised this would take too long to be able to then react to user feedback on the new version within the three year project time frame. We arranged the project into modules so that we could release the software incrementally, distributing a diminishing pool of legacy BoneJ1 plugins alongside a growing set of modernised tools.

We released experimental versions of BoneJ2 in June 2017, where some of the plugins were new, but some were still exactly as they appeared in BoneJ1. With the name experimental we wanted to communicate that while some parts of BoneJ2 were ready to use, the software was still going to go through major changes. For example, we wanted to prepare users for the differences in the look-and-feel of the plugins as they changed from the legacy to modern implementation.

The ambition of moving code to core ImageJ created some challenges. We had to design the algorithms to suit not just our own but everybody's anticipated needs. With this in mind, we tried to adapt the methods to suit as many kinds of images as possible. For example, previously the BoneJ1 plugin Volume Fraction handled only 3D images, whereas its $\mathrm{Op}$ can now process images with any number of dimensions. However, BoneJ2 includes only Area Fraction for 2D images and Volume Fraction for 3D images. With other plugins, such as Connectivity, we found that to support 2D images in addition to 3D images, we would have to develop a significantly different algorithm that would not fit within a single Euler characteristic-calculating Op. Only 3D Connectivity is included in BoneJ2 at present.

\section{Implementation}

Image J and most of the related projects use Git ${ }^{12}$ and GitHub for version management, and Maven for build automation, so those were natural choices for BoneJ2. Features that BoneJ uses have remained free of charge and unencumbered by third-party licence requirements. BoneJ adopts a standard naming scheme for its Git branches, with master containing release-ready code, with new features and bug fixes being developed on topic branches that are merged into master via pull requests.

BoneJ2 is a multi-module Maven project, meaning it consists of several JAVA archive (JAR) packages that reference each other like libraries. That is, they can access each other's public application programming interfaces (APIs) only. Separating functionality into different modules during development makes it more difficult to unintentionally introduce tacit dependencies among modules' code. The multi-module structure helps to organise code, and it makes functionality within BoneJ usable for others without them having to depend on the whole project. As mentioned in the Design section, BoneJ2 is split into Legacy and Modern parts. They are further divided into bonej-legacy-plugins_, bonej-legacy-util_, bonej-plugins, bonej-ops and bonej-utilities. The bonej-ops and bonej-utilities JARs contain code used by the wrapper plugins and could be thought of as the skunkworks of the project where code matures before it is ready to be engineered out of BoneJ and moved to parent projects. As more BoneJ code goes upstream into the core ImageJ platform, this level of structuring will become less necessary. 
Table 1 details the status of all BoneJ1 plugins in relation to BoneJ2. The wrapped external plugins comprise new adaptor code that accesses third-party plugins that had been duplicated in BoneJ1 and subsequently removed from BoneJ2. They contain the small amount of custom functionality, where the BoneJ1 versions of the plugins differed from the originals. In some cases we also contributed to the original plugins. The benefits of not duplicating code are that there is a single upstream project to maintain, whose updates benefit BoneJ with no further downstream work. The secondary function of wrappers is to bundle the plugins into BoneJ, which makes sure they are available to other tools that need them, such as Intertrabecular Angles (ITA). The plugins marked external have no wrappers because they are included in ImageJ by default, and BoneJ1 added no functionality to them.

Ported plugins are fully compatible with the modern ImageJ API and have no legacy dependencies. As much of their functionality as possible has been moved into the ImageJ Ops framework and other parts of the ImageJ platform. While ITA is the only entirely new tool, there are new features in all the ported tools. For example, Connectivity supports images with channels or time in addition to 3 spatial dimensions (hyperstacks), and Anisotropy uses a new method for sampling the image. After modernising the ported external plugins, we submitted them for inclusion in upstream or third-party projects where they fit more naturally. We added the plugins that read custom file formats to SCIFIO (SCientific Image Format Input and Output), so now ImageJ can open them without users needing to install BoneJ. The tools marked legacy are how they appeared in BoneJ1, but their dependencies are managed with Maven.

The pQCT plugins by Timo Rantalainen ${ }^{13}$ were unbundled from BoneJ and placed in their own repository and with their own update site, because the BoneJ team was no longer needed to help with releases, and Rantalainen has always maintained control over the pQCT code and documentation. People who need only the pQCT tools can install them separately without BoneJ, and vice versa. The pQCT update site still includes a plugin for the custom Stratec $p Q C T$ file format even though it is also supported by SCIFIO. This is because SCIFIO is still experimental code, and we wanted to ensure full backwards compatibility.

Discontinued plugins were removed from BoneJ2 altogether. We removed the Structure Model Index (SMI) plugin because it does not measure rods and plates in the presence of the substantial concave curvature that is common in trabecular bone. Such curvature varies as a function of bone volume fraction $(\mathrm{BV} / \mathrm{TV})^{14}$, which can lead to erroneous conclusions about changes in bone architecture. Ellipsoid Factor (EF) ${ }^{15,16}$ replaces BoneJ1's SMI plugin and the early EF prototype Plateness. Due to the risk of misuse, and because it was doing little more for most users than ImageJ's built-in auto-thresholder, we also elected to discontinue Optimise Threshold.

\section{Testing}

During the code overhaul a major effort was made to improve the testing framework and coverage of BoneJ code. We added unit tests to cover more than $90 \%$ of the lines of BoneJ 2 code. Unit tests execute code using standard conditions and check that the expected result is returned. A differing result indicates that something within the tested code, or code that it relies on, has changed and the developers are alerted by the continuous integration tools Maven and Travis. Code with failing unit tests cannot be merged into the master Git branch and thus is prevented from reaching the end user. BoneJ's lower-level code has greater test coverage than higher level code because the unit tests are simpler to write and to include in the continuous integration framework. Testing low-level code thoroughly helps prevent bugs from appearing after implementing more complex software on top of it. BoneJ2 is also included in the SciJava project's testing framework ${ }^{17}$ to ensure that it uses a compatible build environment and compatible versions of dependencies and plugins and so that projects depending on BoneJ2 are also brought up to date. In this way BoneJ2 maintains tested version coordination with the rest of the large ImageJ/Fiji and wider inter-dependent scientific software ecosystem. User interaction testing is partly automated and partly manual. Ideally all tests would be automated, but if for example the code runs a UI call to show a dialog, there is no guarantee that the user will see the dialog. The manual tests are sets of use cases that define a set of repeatable steps of user interaction, and the expected behaviour of BoneJ in response. They are time-consuming, and thus usually performed only after major changes to a plugin.

BoneJ1 plugins were validated against test images during their development, and continuity was ensured by checking that BoneJ2 results matched BoneJ1 results on the same test images. For some plugins results deviate because the technology has changed, for example, the marching cubes algorithm that produces meshes used to calculate surface area is implemented differently in ImageJ Ops than the legacy ImageJ 3D Viewer. As a result, the ratio reported by volume fraction differs by $0.6 \%$ on the sample image bat-cochlea-volume.tif. Mean intercept vectors are generated differently in BoneJ2's Anisotropy than in BoneJ1's Anisotropy, and this introduces a systematic bias to degree of anisotropy calculations that is likely to vary depending on specifics of user images. 


\section{Table 1. Continuity table showing how plugins from BoneJ1 were carried forward.}

\begin{tabular}{|c|c|}
\hline Functionality & Status \\
\hline Analyse Skeleton & wrapped external (Fiji plugin) \\
\hline Anisotropy & ported \\
\hline Connectivity & ported \\
\hline Delete Slice Range & legacy \\
\hline Density Distribution ${ }^{\ddagger \star}$ & ported external (PQCT) \\
\hline Ellipsoid Factor & ported \\
\hline Erode 3D, Dilate 3D & external (ImageJ plugins) \\
\hline Fit Ellipsoid & ported \\
\hline Fit Sphere & legacy \\
\hline Fractal Dimension & ported \\
\hline Help & discontinued \\
\hline Interpolate ROIs & ported external (ImageJ1) \\
\hline Intertrabecular Angles & new \\
\hline Isosurface ${ }^{\star}$ & ported \\
\hline ISQ Reader / Scanco ISQ & ported external (SCIFIO) \\
\hline Kontron IMG & ported external (SCIFIO) \\
\hline Moments of Inertia & legacy \\
\hline Neck Shaft Angle & discontinued \\
\hline Optimise Threshold & discontinued \\
\hline Orientation & legacy \\
\hline Particle Analyser & legacy \\
\hline Plateness & discontinued \\
\hline Purify & legacy \\
\hline Stratec pQCT & ported external (SCIFIO \& PQCT) \\
\hline Skeletonise 3D & wrapped external (Fiji plugin) \\
\hline Slice Geometry & legacy \\
\hline Structure Model Index & discontinued \\
\hline Thickness & wrapped external (Fiji plugin) \\
\hline Usage reporting & ported \\
\hline Volume Fraction ${ }^{\dagger}$ & ported \\
\hline \multicolumn{2}{|l|}{$\star$ Renamed to Surface area } \\
\hline \multicolumn{2}{|c|}{ tSplit into Surface fraction and Area/Volume fraction } \\
\hline \multicolumn{2}{|c|}{ ¥Added to BoneJ1 after the publication of Doube et al. (2010) ${ }^{1}$} \\
\hline \multicolumn{2}{|c|}{ *Split into an independent plug-in } \\
\hline
\end{tabular}




\section{Continuous integration and deployment}

User builds of BoneJ2 are deployed after running all tests, updating the Maven artefact version numbers, Git tagging the commits and archiving a reference copy of the tagged code with a digital object identifier (DOI) at Zenodo (project doi:10.5281/zenodo.142726218). The latest Maven artefacts, which are specific versions of the BoneJ JAR files, are uploaded to BoneJ's ImageJ update site. Users are then reminded by the ImageJ updater to update their plugins. Plugin updates and their dependencies are listed by the updater, which retrieves them from the update site. Updated plugins become active after restarting ImageJ.

Since v7.0.0 (styloid) BoneJ has been in a high-frequency release cycle, improving performance, fixing bugs, and adding small user features, and incrementing the patch version according to semantic versioning conventions. BoneJ2's multi-module design means that for most of the minor and patch releases at least one of the modules contains no functional code changes. The semantic versioning relates to the BoneJ2 project as a whole and not to individual modules' jar files, resulting in frequent 'empty' updates of jar files especially for the minor and patch revisions. Originally the release and versioning of each module was handled separately, but that proved too arduous in the long run.

\section{Operation}

The minimal system requirements are:

- Windows, Mac OS X, GNU/Linux or other OS running Java 8 or later.

- $\quad$ ImageJ2 with the java-8 and with or without the Fiji update site enabled.

- Minimal hardware requirements are readily satisfied by a contemporary laptop computer, however, performance will scale with increasing CPU cores and RAM especially for operations running in multiple threads and on large data sets.

The tools in BoneJ are specialised and require a working knowledge of image processing. The purpose of developing BoneJ as an ImageJ plugin is to have ready access to its large ecosystem of filtering, segmentation, and thresholding tools for image preprocessing. Most BoneJ algorithms expect binary images, which have only two values: one for background and one for foreground. In BoneJ's case the foreground is assumed to represent bone. It is the user's responsibility to segment and threshold their images so that the foreground truly corresponds to bone, and not soft tissue, noise, or other imaging artefacts. The plug-ins do not and cannot determine if the binary images represent the samples adequately. Users are encouraged to run sensitivity analyses to determine how reasonable variations to their processing and analysis settings affect readout values. They should also check BoneJ's output using test images, which could be synthetic images with known properties or images that represent well-characterised test objects with features relevant to the user's research domain.

\section{Use cases}

In this example, we calculate bone volume fraction (BV/TV), ellipsoid factor (Tb.EF), degree of anisotropy (DA), thickness (Tb.Th), separation (Tb.Sp), and connectivity density (Conn.D) of trabecular bone from the femoral head of Apteryx haastii, using the interactive menu-driven approach and an ImageJ macro. Variable parameters of all the plugins are documented at https://imagej.github.io/plugins/bonej. As a comparison, the same parameters were calculated with BoneJ 1.4.3, using 'Auto Mode' for Anisotropy

\section{Interactive use case}

1. Install BoneJ according to the instructions at https://imagej.github.io/plugins/bonej\#installation

2. Download umzc_378p_Apteryx_haastii_head.tif.bz2 from doi:10.6084/m9.figshare.7257179

3. Extract umzc 378p_Apteryx haastii head.tif from the .bz2 file. Note that it is a 16-bit greyscale X-ray microtomography image with isotropic pixel spacing $(10.1 \mu \mathrm{m})$, and that the trabecular bone and bone marrow fill the entire image volume.

4. Run Plugins $>$ Macros $>$ Record... to capture the sequence of commands

5. Run Process $>$ Filters $>$ Gaussian Blur (3D) with a sigma of 2.0 in all 3 dimensions to smooth bone surfaces

6. Run Image > Adjust $>$ Threshold, select Dark background and Stack histogram, click Auto and then click Apply

7. In the Convert Stack to Binary window, unselect all checkboxes and select Method: Default, Background: Dark, click OK 
8. Pass a cursor over the image and check in the ImageJ status bar that the pixel value for trabeculae is 255 and marrow space 0. The inverting lookup table (LUT) indicates that foreground is displayed as black and background white.

9. Run Plugins $>$ BoneJ $>$ Anisotropy and select Recommended minimums, click OK to calculate DA

10. Run Plugins $>$ BoneJ $>$ Fraction $>$ Area/Volume Fraction to calculate BV/TV

11. Run Plugins > BoneJ > Ellipsoid Factor with Vectors:100, Sampling Increment: 0.435, Skeleton points per ellipsoid:10, Contact sensitivity:1, Maxmimum iterations: 50, Maximum drift:1.73, Minimum semi-axis: 1, Repetitions: 3, Average over largest $n$ ellipsoids: 3, Seed points based on distance ridge: true, Threshold for distance ridge: 0.6, Seed points for topology preserving skeletonisation: false, Show Flinn plots: false; Show algorithm convergence, false; Show verbose output images, false, to calculate Tb.EF

12. Run Plugins $>$ BoneJ $>$ Purify to remove small background particles

13. Run Plugins $>$ BoneJ $>$ Connectivity (Modern) to calculate Conn.D

\section{Scripting use case}

Use the macro recorded from the steps above, edited to the below, taking care to place each run () command on a single line without any carriage returns. It is also possible to incorporate BoneJ plugins in Python scripts.

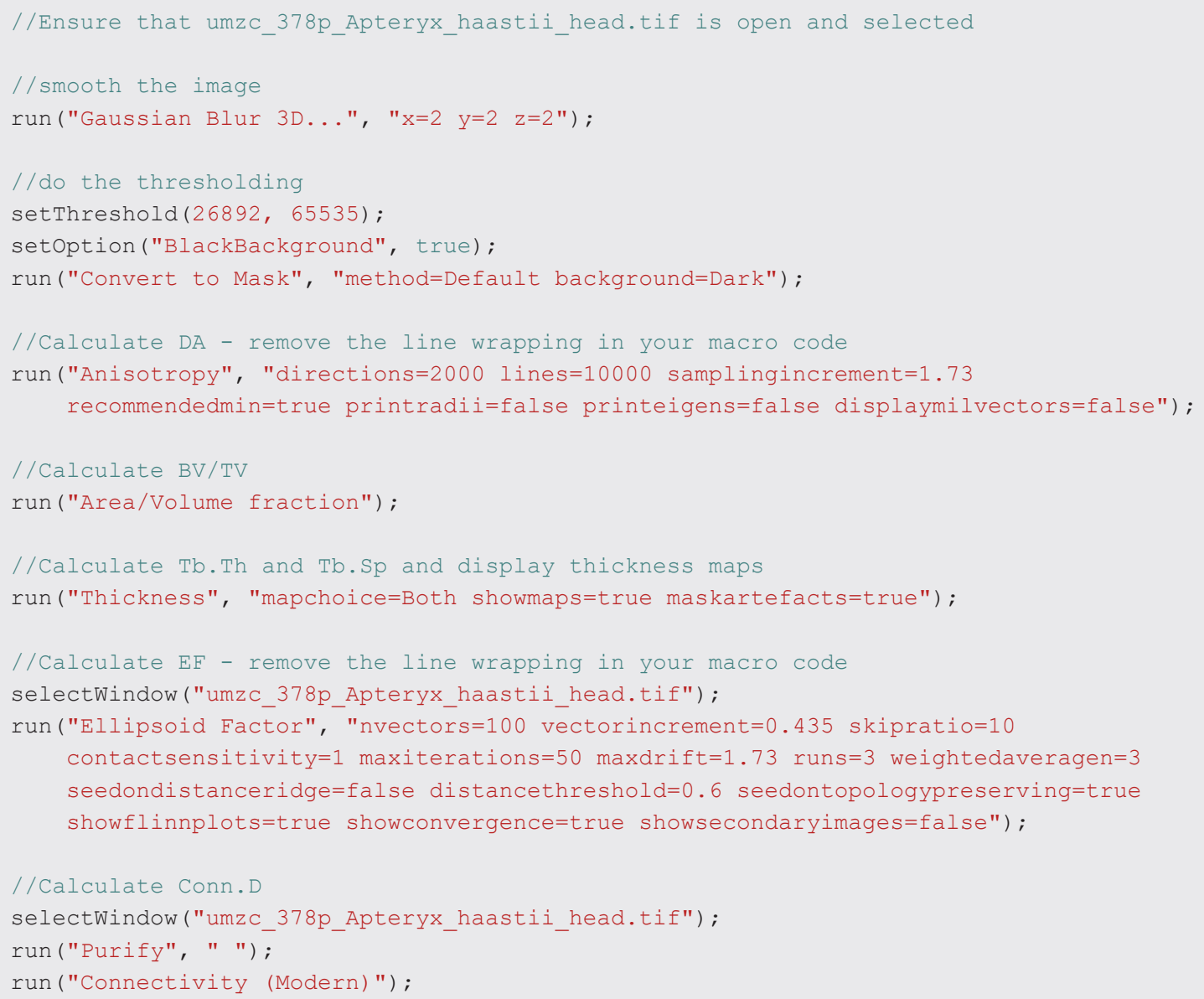

\section{Use case results}

Both interactive and scripted examples log numerical results to the BoneJ Result table (Table 2) and produce output images that may be used for further analysis and to visualise the spatial distribution of measurements (Figure 2). 


\begin{abstract}
Table 2. Expected results logged in the BoneJ result table comparing BoneJ2 and BoneJ1 output. Due to the stochastic nature of sampling both degree of anisotropy (DA) and ellipsoid factor (EF) vary between runs in an image- and setting-specific manner. Modern Anisotropy and Ellipsoid Factor ${ }^{16}$ plugins differ in implementation from those in BoneJ1, so some minor variation in output is expected. Users are strongly encouraged to run sensitivity analyses. to select settings for Anisotropy and Ellipsoid Factor (as we have done) ${ }^{16}$ that produce a stable result on their images. Note that Thickness, Volume Fraction and Connectivity are direct ports from BoneJ1 to BoneJ2 and have no stochastic character, so their results are expected to be invariant for the same input image. BoneJ2's Purify uses Particle Analyser's improved ConnectedComponents code ${ }^{19}$ and completes in $\sim 1 \mathrm{~s} \mathrm{compared}$ to $\sim 15 \mathrm{~s}$ in Bonej1.
\end{abstract}

\begin{tabular}{|c|c|c|}
\hline Image & $\begin{array}{l}\text { BoneJ2 } \\
\text { (styloid-r11) }\end{array}$ & BoneJ 1.4.3 \\
\hline DA & 0.51675 & 0.56665 \\
\hline $\mathrm{BV}\left(\mathrm{mm}^{3}\right)$ & 25.92792 & 25.92792 \\
\hline $\mathrm{TV}\left(\mathrm{mm}^{3}\right)$ & 62.21983 & 62.21983 \\
\hline BV/TV & 0.41671 & 0.41671 \\
\hline Tb.Th Mean (mm) & 0.21246 & 0.21246 \\
\hline Tb.Th Std. Dev. (mm) & 0.06347 & 0.06347 \\
\hline Tb.Th Max (mm) & 0.43277 & 0.43277 \\
\hline Tb.Sp Mean (mm) & 0.47014 & 0.47014 \\
\hline Tb.Sp Std. Dev. (mm) & 0.14888 & 0.14888 \\
\hline Tb.Sp Max (mm) & 0.96242 & 0.96242 \\
\hline Median EF & -0.14632 & $\mathrm{NaN}$ (mean, -0.21451) \\
\hline Max EF & 0.91779 & 0.91695 \\
\hline Min EF & -0.84867 & -0.93064 \\
\hline Euler $\operatorname{char}(\chi)$ & -291 & -291 \\
\hline Corr. Euler $(\chi-\Delta \chi)$ & -238.375 & -238.375 \\
\hline Connectivity & 239.375 & 239.375 \\
\hline Conn.D $\left(\mathrm{mm}^{3}\right)$ & 3.84725 & 3.84725 \\
\hline
\end{tabular}

\title{
Developer use case
}

New BoneJ developers are encouraged to read the extensive developer documentation at BoneJ's GitHub wiki. New developers are welcome to fork the repository, make and commit changes to their personal fork, and then submit a pull request (PR) to bonej-org/BoneJ2 for review and merging by the BoneJ team.

\section{Discussion}

BoneJ2 made its first general release (semantic version 7.0.0, codenamed styloid) via the ImageJ updater in March 2020, and events with Modern versions began appearing with greater frequency in the usage report, with 1,000 - 4,000 events recorded weekly from 190 cities in 36 countries between March and June 2020.

\section{Limitations}

During the experimental release phase, we noticed that naïve direct ports of BoneJ1 code to BoneJ2 using ImgLib2 could result in significant performance degradation, despite Rueden et al.'s claims of minimal performance loss or even performance enhancement using an Ops design ${ }^{8}$. By instrumentation with timer code, 


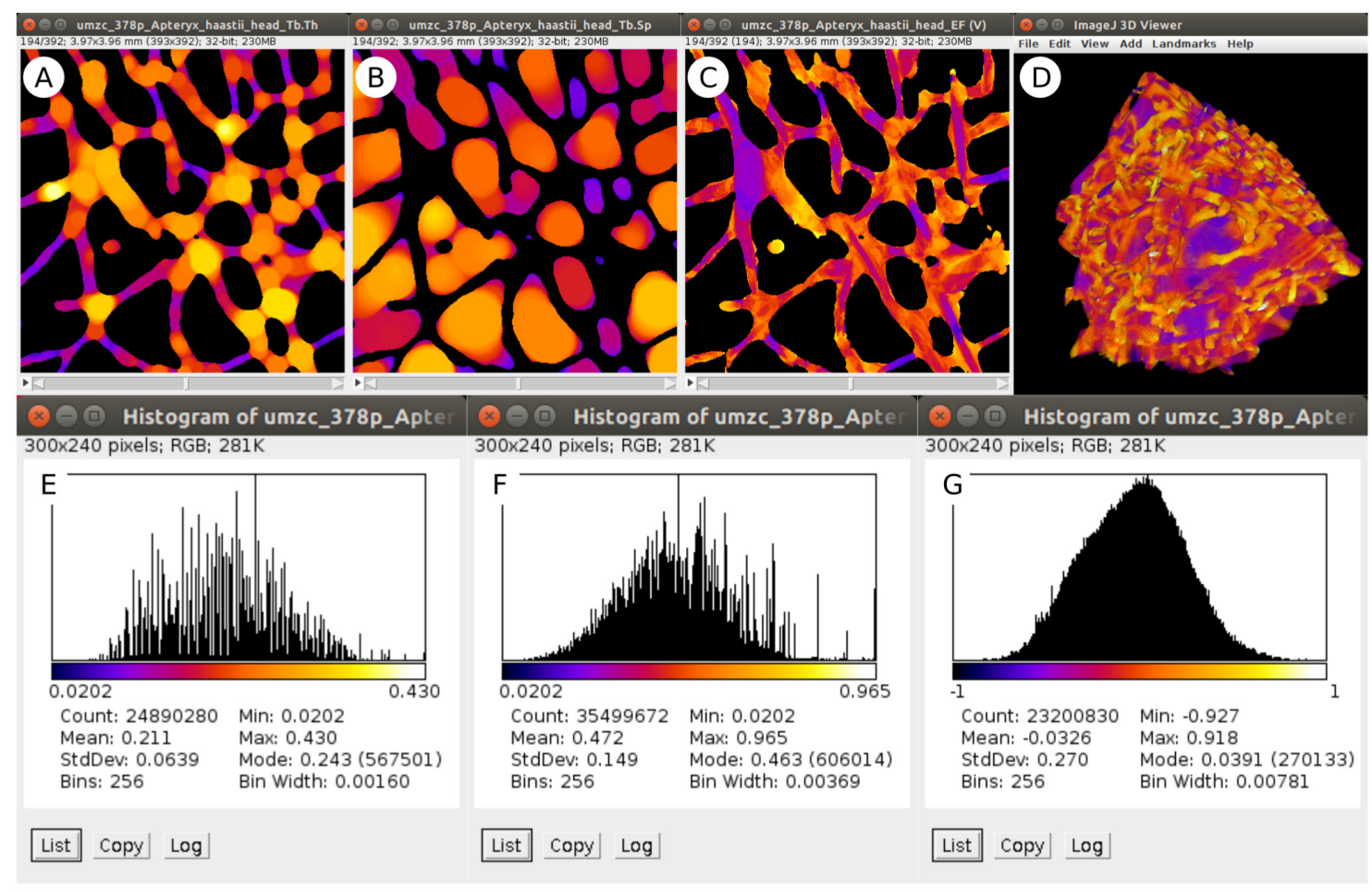

Figure 2. Sample image output from the worked example above. Stacks displaying maps of Tb.Th (A), Tb.Sp (B), Tb.EF (C) contain pixels whose values represent the local thickness, separation or ellipsoid factor respectively, each with the Fire lookup table (LUT) applied and spread between the minimum and maximum pixel value. Background pixels are set to NaN (not a number) to exclude them from numerical analysis. Plotting histograms (Analyze > Histogram...) of the output image stacks results in distributions and summary statistics for Tb.Th (E), Tb.Sp (F) and Tb.EF (G): note the relation between the LUT and histogram $x$-axis values. Converting the EF image (C) to RGB (Image > Type $>$ RGB) and loading in the 3D Viewer (Plugins > 3D Viewer) results in an interactive 3D visualisation (D).

and inspection of hotspots with a profiler (VisualVM, Oracle), we found that use of ImgLib2's RandomAccess, setPosition() and get() methods can be very costly in computation resources compared to accessing pixel values from primitive arrays. Even after implementing multithreading and avoiding RandomAccess with a sequential pixel access design, Modern Connectivity (which uses a cursor) is about half the speed of Legacy Connectivity, which uses primitive array access to read pixel values. The conscious design choice to make pixel array access difficult with ImgLib2's libraries to nudge developers to handle $N$-dimensional images can have the unfortunate side effect of making pixel access slow. Future BoneJ developments will balance the potential for generalisation of algorithms into $\mathrm{N}$-dimensional images versus execution performance. Algorithms that function exclusively in 3 spatial dimensions will continue to use higher performance primitive array access rather than the slower $\mathrm{N}$-dimensional approach of ImgLib2.

We were also held back by the realisation of new core ImageJ functionality lagging the proposals of the functionality by several years. We intended to incorporate sophisticated region of interest (ROI) handling in BoneJ2, but libraries to do so were not developed by the ImageJ team. In that regard, we have adopted a less ambitious practice of developing against currently available technologies and integrating new third-party technologies only after they are released to users. In retrospect we could have released the first experimental version earlier to make it easier for users to adopt the new software.

The multi-module strategy allowed earlier releases and feedback but made the software more complex to manage because legacy code needs to be kept separate from modern code, which creates some duplicate work in the construction and distribution of BoneJ. The separation of concerns in BoneJ2's engineering is intended to allow efficient adoption of new functionality but spreading the implementation across the ImageJ software stack has made the design of BoneJ more complicated. We must compile and build multiple components, and ensure they work together, before we can safely release a BoneJ version. We must also ensure that the changes we make to 
ImageJ (or other external code) are accepted, merged, and released before we can make the latest BoneJ available. On occasion we have had to revise our code that was already accepted into ImageJ to implement a detail that a BoneJ wrapper plugin required.

Future

BoneJ will continue to be maintained and developed as opportunities arise to improve research tools.

\section{Funding}

BoneJ's development was initially supported as an unanticipated outcome of a BBSRC project grant (BB/F001169/1). The strategic value of software resources was recognised by MD while working as a postdoc, leading to the publication of the software and its paper and development of the initial project resources. Subsequent maintenance was performed ad-hoc as a service to the community alongside other job responsibilities but was not directly funded until a successful bid to Wellcome Trust (108442/Z/15/Z), after a similar bid to BBSRC was declined. BBSRC subsequently funded some development on BoneJ's Ellipsoid Factor that was described as part of an ordinary project grant (BB/P006167/1).

In November 2019, in recognition of the need for specific funding for software and other technology development for the biosciences, Wellcome Trust split the Biomedical Resource and Technology Development Grant scheme that funded BoneJ2 into two parts, ringfencing funds for Technology Development Grants to "substantially enhance an existing technology or resource" 20 . Ongoing maintenance of existing resources including the day-to-day servicing of user requests would appear not to satisfy the condition of "substantial enhancement" which could contribute to newly upgraded tools being abandoned due to lack of dedicated support personnel.

Larger institutions (such as AAF's current employer UCL) may decide to set up a core facility with central funds and/or accounting processes to recoup costs from researchers' grants, to support research software engineers who service members of the institution's research community. Embedding RSEs within individual research groups remains challenging due to RSEs needing specialist skills including research experience, yet funding schemes often require hiring a technician or postdoctoral scientist which are roles distinct from RSE. Institutions may also lack a suitable career structure that encapsulates RSEs' pay, role, responsibilities, and expected output as service staff, rather than research staff $^{21}$. Professional Research Software Engineering societies have been established in (at least) the UK, US, Netherlands, Germany, and the Nordic countries, while the UK's Software Sustainability Institute and UKRI e-infrastructure roadmap are actively highlighting these weaknesses in the funding and career landscape for individuals who may be interested in pursuing an RSE, rather than classical academic, career pathway. Given limited personnel funds available in many grant schemes, principal investigators may prefer to recruit an 'all-rounder' postdoctoral researcher who can code in addition to performing research. Our experience as a small project team with a PI (MD), RSE (RD), and PhD student then postdoc (AAF) highlighted the added value that a person with an engineering and service orientation brings to a scientific team. The BoneJ2 project also demonstrated the labour intensiveness of quality engineering practice which cannot be expected of a postdoctoral researcher, who is judged mainly on their academic writing output. The RSE is responsible for setting up and maintaining development practices that lead to more robust and reliable programs and user experience, which is vital when software is disseminated to other users, and when the project receives contributions from third parties. The RSE insists on good development practices, so that scientists' new approaches to problems can be packaged in a manner suitable for public consumption, and in doing so enhance the impact and societal value of research.

\section{Summary}

How functionality is achieved on the development side is a secondary concern for users, whose primary concern is getting results from data. Poor software development practice may not affect users' experience of the software, but fast and undisciplined coding is likely to lead to more bugs and harder to solve errors. In the long run it may also become increasingly difficult to add new features and respond to changes. On the other hand, researchers are usually under time pressure to produce scientific results with little reason to dedicate the effort required to write sustainable software. An important part of writing maintainable code is to make it easy to read and understand. We posit that code clarity is especially important for research software, because researchers read program code to understand and adapt the methodology of an experiment, making code an essential part of the scientific record. A counterintuitive outcome of working on community software like BoneJ is that the more useful contributions are removed from the project and donated to the common pool upstream, which creates a need for developers to let go of ownership. Retaining code ownership for reasons of maintaining personal key performance indicators may be counterproductive to generating lasting impact from development work. BoneJ 
remains an open-source project with all changes and much of the discussion about its development occurring in the public domain; we consider process transparency an essential feature of scientific discourse.

\section{Data availability}

All data underlying the results are available as part of the article and no additional source data are required.

\section{Software availability}

1. Software available from: https://bonej.org/ and https://imagej.github.io/plugins/bonej

2. Source code available from: https://github.com/bonej-org/BoneJ2

3. Archived source code at time of publication: https://doi.org/10.5281/zenodo.1427262 18

4. Licence: Simplified BSD-2 licence

\section{Author contributions}

RD wrote the BoneJ2 code, set up its development infrastructure and drafted the manuscript. AAF wrote the code and drafted the manuscript. MD conceived the project, wrote BoneJ1 and BoneJ2 code, and drafted the manuscript.

\section{Acknowledgements}

We thank Curtis Rueden, Johannes Schindelin, Mark Hiner, Jan Eglinger, Kevin Eliceiri, Kyle Harrington, Ulrich Gunther, Timo Rantalainen, Ignacio Arganda-Carreras, Karl-Heinz Kunzelmann, Per Christian Henden, Jens Bache-Wiig, Natalie Reznikov, the Software Sustainability Institute, and our many users who contribute questions on the ImageJ Forum.

1. Doube M, Kłosowski MM, Arganda-Carreras I, et al.: Bonej: free and extensible bone image analysis in Imagej. Bone. 2010; 47(6): 1076-1079.

PubMed Abstract | Publisher Full Text | Free Full Text

2. Schneider CA, Rasband WS, Eliceiri KW: NIH Image to ImageJ: 25 years of image analysis. Nat Methods. 2012; 9(7): 671-675. PubMed Abstract | Publisher Full Text | Free Full Text

3. Doube M, Kłosowski MM, Wiktorowicz-Conroy AM, et al.: Trabecular bone scales allometrically in mammals and birds. Proc Biol Sci. 2011; 278(1721): 3067-3073.

PubMed Abstract | Publisher Full Text | Free Full Text

4. Doube M, Yen SCW, Kłosowski MM, et al.: Whole-bone scaling of the avian pelvic limb. J Anat. 2012; 221(1): 21-29. PubMed Abstract | Publisher Full Text | Free Full Text

5. Carriero A, Doube M, Vogt M, et al.: Altered lacunar and vascular porosity in osteogenesis imperfecta mouse bone as revealed by synchrotron tomography contributes to bone fragility. Bone. 2014; 61: 116-124.

PubMed Abstract | Publisher Full Text

6. Parfitt AM, Drezner MK, Glorieux FH, et al.: Bone histomorphometry: standardization of nomenclature, symbols, and units. Report of the ASBMR Histomorphometry Nomenclature Committee. J Bone Miner Res. 1987; 2(6): 595-610. PubMed Abstract | Publisher Full Text

7. Schindelin J, Arganda-Carreras I, Frise E, et al.: Fiji: an open-source platform for biological-image analysis. Nat Methods. 2012; 9(7): 676-682.

PubMed Abstract | Publisher Full Text | Free Full Text

8. Rueden $\mathrm{CT}$, Schindelin J, Hiner MC, et al.: ImageJ2: ImageJ for the next generation of scientific image data. BMC Bioinformatics. 2017; 18(1): 529

PubMed Abstract | Publisher Full Text | Free Full Text

9. Schmid B, Schindelin J, Cardona A, et al.: A high-level 3D visualization API for Java and ImageJ. BMC Bioinformatics. 2010; 11: 274.

PubMed Abstract | Publisher Full Text | Free Full Text

10. Arganda-Carreras I, Fernández-González R, Muñoz-Barrutia A, et al:: 3D reconstruction of histological sections: Application to mammary gland tissue. Microsc Res Tech. 2010; 73(11): 1019-1029. PubMed Abstract | Publisher Full Text
11. Katz DS, McHenry K, Reinking C, et al.: Research Software Development \& Management in Universities: Case Studies from Manchester's RSDS Group. Illinois' NCSA, and Notre Dame's CRC. In 2019 IEEE/ACM 14th International Workshop on Software Engineering for Science (SE4Science). 2019; 17-24. Publisher Full Text

12. Torvalds L, Hamano J: git. 2016. Reference Source

13. Rantalainen T, Nikander R, Heinonen A, et al:: An open source approach for regional cortical bone mineral density analysis. J Musculoskelet Neuronal Interact. 2011; 11(3): 243-248. PubMed Abstract

14. Salmon PL, Ohlsson C, Shefelbine SJ, et al.: Structure model index does not measure rods and plates in trabecular bone. Front Endocrinol (Lausanne). 2015; 6: 162 PubMed Abstract | Publisher Full Text | Free Full Text

15. Doube M: The ellipsoid factor for quantification of rods, plates, and intermediate forms in 3D geometries. Front Endocrinol (Lausanne). 2015; 6: 15.

PubMed Abstract | Publisher Full Text | Free Full Text

16. Felder AA, Monzem S, De Souza R, et al.: The plate-to-rod transition in trabecular bone loss is elusive. bioRxiv. 2021 2020.05.14.081042 Publisher Full Text

17. Rueden C: scijava/pom-scijava. 2021. Reference Source

18. Doube M, Domander R, Rueden C, et al:: bonej-org/BoneJ2: styloid-r11 (Version bonej-7.0.11). Zenodo. 2021. http://www.doi.org/10.5281/zenodo.4635373

19. Doube M: Multithreaded two-pass connected components labelling and particle analysis in ImageJ. Roy Soc Open Sci. 2021 8(3): 201784.

Publisher Full Text

20. Wellcome Trust: Biomedical Resource and Technology Development Grants: update I Wellcome. 2019. Reference Source

21. Tsang E, Allard T, Wilson B, et al:: CW20 speed blog: How do we persuade funders to support software maintenance? Software Sustainability Institute, June 2020. Reference Source 


\section{Open Peer Review}

\section{Current Peer Review Status:}

\section{Version 2}

Reviewer Report 04 May 2021

https://doi.org/10.21956/wellcomeopenres.18585.r43626

(C) 2021 Madi $\mathrm{K}$ et al. This is an open access peer review report distributed under the terms of the Creative Commons Attribution License, which permits unrestricted use, distribution, and reproduction in any medium, provided the original work is properly cited.

\section{Loic Courtois}

3Dmagination Ltd, Didcot, UK

Kamel Madi

3Dmagination Ltd, Didcot, UK

The authors have answered all my comments.

Competing Interests: No competing interests were disclosed.

Reviewer Expertise: X-ray computed tomography, advanced image processing, plugins development.

We confirm that we have read this submission and believe that we have an appropriate level of expertise to confirm that it is of an acceptable scientific standard.

Reviewer Report 28 April 2021

https://doi.org/10.21956/wellcomeopenres.18585.r43625

(C) 2021 Kersh M. This is an open access peer review report distributed under the terms of the Creative Commons Attribution License, which permits unrestricted use, distribution, and reproduction in any medium, provided the original work is properly cited.

\section{Mariana Kersh}

${ }^{1}$ Mechanical Science and Engineering, University of Illinois at Urbana-Champaign, Urbana, IL, USA

2 Beckman Institute for Advanced Science and Engineering, University of Illinois at UrbanaChampaign, Urbana, IL, USA

I think the inclusion of the previous version outputs to the current BoneJ2 output data helps to demonstrate that the implementation of the new structure for BoneJ2 has been done 
properly/doesn't result in significant changes. This will certainly give the community confidence when making the transition to BoneJ2.

Thank you again for your contributions.

Competing Interests: No competing interests were disclosed.

Reviewer Expertise: Musculoskeletal biomechanics. Structure-function in bone and passive tissues.

I confirm that I have read this submission and believe that I have an appropriate level of expertise to confirm that it is of an acceptable scientific standard.

\section{Version 1}

Reviewer Report 21 April 2021

https://doi.org/10.21956/wellcomeopenres.18322.r42731

(C) 2021 Schmidt D. This is an open access peer review report distributed under the terms of the Creative Commons Attribution License, which permits unrestricted use, distribution, and reproduction in any medium, provided the original work is properly cited.

\section{Deborah Schmidt}

Max Delbrück Center for Molecular Medicine in the Helmholtz Association, Bonn, Germany

The publication Bonej2 - refactoring established research software captures the effort of maintaining and modernizing research software while adjusting to infrastructural changes in the community and ensuring backwards compatibility. The authors depict in detail the longstanding process of moving the ImageJ plugin suite for skeletal biology image analysis first released in 2010 into the ImageJ2 / SciJava ecosystem. The approach described in the paper to refactor the existing code base in an organized fashion can serve as a blueprint for other projects to follow their footsteps, i.e. by clearly separating plugins into legacy and modern components, a high percentage of unit test coverage, and analyzing and documenting analysis divergences after refactoring plugins.

The design was adjusted to the modularity of SciJava by creating independent, reusable pieces and I highly commend the efforts made to move general purpose code into the Imagej2 / SciJava core libraries such as imagej-ops and SCIFIO. Both SCIFIO as well as ImgLib2 deserve to be referenced though.

The paper is educating for research groups maintaining software, mentioning the challenges of the ecosystem and the amount of work that isn't achievable for a scientist in part-time, but was executed by a dedicated Research Software Engineer. Same applies to the depicted funding challenges like changes to the Wellcome Trust seemingly hindering the ongoing financial support of maintaining Bonej2. 
Strategies to ease the user into switching to new implementations of plugins are mentioned. It would be valuable to read about any feedback the authors might have received from their users regarding the refactoring process. Since the usage of opt-in Google analytics was mentioned, insightful statistics could be included.

Is the rationale for developing the new software tool clearly explained?

Yes

Is the description of the software tool technically sound?

Yes

Are sufficient details of the code, methods and analysis (if applicable) provided to allow replication of the software development and its use by others?

Yes

Is sufficient information provided to allow interpretation of the expected output datasets and any results generated using the tool?

Yes

Are the conclusions about the tool and its performance adequately supported by the findings presented in the article?

Yes

Competing Interests: No competing interests were disclosed.

Reviewer Expertise: Software engineering, molecular biology.

I confirm that I have read this submission and believe that I have an appropriate level of expertise to confirm that it is of an acceptable scientific standard.

Reviewer Report 13 April 2021

https://doi.org/10.21956/wellcomeopenres.18322.r42735

(C) 2021 Kersh M. This is an open access peer review report distributed under the terms of the Creative Commons Attribution License, which permits unrestricted use, distribution, and reproduction in any medium, provided the original work is properly cited.

\section{Mariana Kersh}

${ }^{1}$ Mechanical Science and Engineering, University of Illinois at Urbana-Champaign, Urbana, IL, USA

2 Beckman Institute for Advanced Science and Engineering, University of Illinois at UrbanaChampaign, Urbana, IL, USA

This paper describes the rationale and methods used to update BoneJ - an open-source plug-in of ImageJ which is widely used within the bone community (as well as other research areas making use of morphometric analyses of structures). One motivation for this work was the update of 
Image to ImageJ2 which resulted in third party libraries, which BoneJ relied on, being deleted or no longer maintained.

The description of these changes and the subsequent enormous amount of work involved in adapting to this is well described at a high-level and very much appreciated. The call for more transparency in science in general includes software methods. I congratulate the authors on their contributions towards this cause and applaud their call to attention to the dilemma they (and others) face that choose to contribute to an effort that is needed by the community but usually not recognized for advancement career-wise. This is something our community needs to address and is an important component of this paper.

With respect to their development efforts for BoneJ2, my background is not in software development, so I write this review as an "informed user".

If the first purpose of this paper is to the updates to Bone 2 that ensure compatibility with Imagej2/Fiji, it would be helpful to see a few side-by-side examples or other performance metrics. For example, in the use case shown- it's not clear to me how I should interpret these results (Table 2, Figure 2). What is the "expected" result? Would it make sense to compare this to the legacy structure to demonstrate that the modernized structure of the plug-in still functions properly and/or with similar processing times, etc. I understand that the way the data is sampled for some of the metrics may result in some differences, but overall, the values should still be quite close. A second option to demonstrate robustness would be to report the standard deviations (or range) of results between repeated runs on the same dataset.

Thanks again to the developers for their contributions to an important tool. I hope others, including funding agencies and senior academics that evaluate career progress, recognize the value of such contributions.

Is the rationale for developing the new software tool clearly explained?

Yes

Is the description of the software tool technically sound?

Yes

Are sufficient details of the code, methods and analysis (if applicable) provided to allow replication of the software development and its use by others?

Yes

Is sufficient information provided to allow interpretation of the expected output datasets and any results generated using the tool?

Partly

Are the conclusions about the tool and its performance adequately supported by the findings presented in the article?

Partly

Competing Interests: No competing interests were disclosed. 
Reviewer Expertise: Musculoskeletal biomechanics. Structure-function in bone and passive tissues.

\section{I confirm that I have read this submission and believe that I have an appropriate level of expertise to confirm that it is of an acceptable scientific standard, however I have significant reservations, as outlined above.}

Author Response 21 Apr 2021

Michael Doube, The Royal Veterinary College, University of London, London, UK

We thank the reviewer for their understanding and support of this manuscript.

If the first purpose of this paper is to the updates to Bone/2 that ensure compatibility with ImageJ2/Fiji, it would be helpful to see a few side-by-side examples or other performance metrics. For example, in the use case shown-it's not clear to me how I should interpret these results (Table 2, Figure 2). What is the "expected" result? Would it make sense to compare this to the legacy structure to demonstrate that the modernized structure of the plug-in still functions properly and/or with similar processing times, etc. I understand that the way the data is sampled for some of the metrics may result in some differences, but overall, the values should still be quite close. A second option to demonstrate robustness would be to report the standard deviations (or range) of results between repeated runs on the same dataset.

We have added a column to table 2 that includes the results from Bone 1.4.3 (the final release of BoneJ1) after running the same interactive use case, showing that the nonstochastic plugins produce identical results in legacy and modern implementations.

The stochastic plugins that rely on random sampling Anisotropy and Ellipsoid Factor have had some improvements made to their implementations between BoneJ1 and BoneJ2 so we don't expect a precise reproduction of DA and EF values, however, they should be close.

We add some description of Table 2 to the text to aid the reader in their interpretation of the results and some further description of e.g. improvements to Purify's execution speed. We also direct the reader to a new publication on Ellipsoid Factor in which thorough sensitivity analyses were performed, and encourage readers to do sensitivity analysis (running the analysis repeatedly with a range of reasonable input settings and observing the variation in output) as a matter of course when developing their image processing pipelines.

It is important to emphasise that in testing and for validation of correct function, simple test images with well-known properties are used to ensure that the new code isn't simply repeating the mistakes of old code to get the same result. Instead both sets of code must independently calculate the correct (expected) result from the same image. The example image provided for the use case is too complicated to use for validation in a meaningful way because it is not obvious, a priori, what the precise results should be. It is, however, useful for demonstrating real-world performance in this comparison of old and new code.

Competing Interests: No competing interests were disclosed. 
Reviewer Report 18 March 2021

https://doi.org/10.21956/wellcomeopenres.18322.r42734

(C) 2021 Madi K et al. This is an open access peer review report distributed under the terms of the Creative Commons Attribution License, which permits unrestricted use, distribution, and reproduction in any medium, provided the original work is properly cited.

\section{Loic Courtois}

3Dmagination Ltd, Didcot, UK

Kamel Madi

3Dmagination Ltd, Didcot, UK

The article raises an important concern about Bonej2 longevity and open source software in general. The key challenges and technical steps taken to improve the quality of Bonej code are well described in the introduction. The efforts made by the authors and the process of transparency are greatly appreciated due to the difficult task undertaken to make BoneJ2 a robust tool. The progressive transition from legacy to modern architecture is nicely detailed; the description is very technical though (Design/Implementation section) which is fine for an audience like developers or RSE but could be difficult to comprehend from a "user" point of view. From a user point of view, (i) the interactive use case and the expected results are very useful to test the plugin and validate the results, (ii) the limitations of the new implementation, and in particular accessing pixel values, will be useful information to the community during the transition period. The authors have done a great job at raising awareness on the importance of funding, training and crucial role played by permanent staff such as RSE to maintain the code. The paper could help setting examples that others could follow and help growing concern and giving more resources to teams working on open source packages such as BoneJ.

Comments/suggestions:

1. Interactive use case: step 11 did not work for me. After running the ellipsoid factor plugin, looks like something is running but never ends.

2. Could be interesting to validate the results provided in the use case against another software that uses a similar method (focus on just trabecular thickness for example).

Is the rationale for developing the new software tool clearly explained?

Yes

Is the description of the software tool technically sound?

Yes

Are sufficient details of the code, methods and analysis (if applicable) provided to allow replication of the software development and its use by others?

Yes

Is sufficient information provided to allow interpretation of the expected output datasets 
and any results generated using the tool?

Yes

Are the conclusions about the tool and its performance adequately supported by the findings presented in the article?

Yes

Competing Interests: No competing interests were disclosed.

Reviewer Expertise: X-ray computed tomography, advanced image processing, plugins development.

We confirm that we have read this submission and believe that we have an appropriate level of expertise to confirm that it is of an acceptable scientific standard.

Author Response 19 Apr 2021

Michael Doube, The Royal Veterinary College, University of London, London, UK

Thank you for the comments and support.

1. Interactive use case: step 11 did not work for me. After running the ellipsoid factor plugin, looks like something is running but never ends.

There is a typo in the Vectors setting, which should be 100, not 1, and is fixed in the resubmission. Each ellipsoid is represented as a set of unit vectors multiplied by the ellipsoid's $3 \times 3$ matrix. Collisions between the ellipsoid and the foreground/background boundary are calculated for each of the unit vectors to determine whether or not it is fully contained within foreground. So while it is not strictly illegal to use only 1 vector, the sampling is too sparse to have much meaning. We have found that 100 is a reasonable compromise between sampling density and efficiency, and believe there are more efficient sampling methodologies to discover than the one employed so far.

2. Could be interesting to validate the results provided in the use case against another software that uses a similar method (focus on just trabecular thickness for example).

Comparisons with other software have been performed before on Bonej's algorithms (e.g. EF vs SMI vs SMI in https://doi.org/10.3389/fendo.2015.00162) but are not really validation. Validation requires that a ground truth or expected result is known. In 'validating' against other software, one is making an assumption that the other software has no faults and makes a similar representation of the data as the software under test. It is possible that both sets of software get the same wrong answer, for example, or get the same answer by chance rather than design. If they get different answers there is no way to tell which (if any) of them got the right answer without reference to ground truth. If the other software has been validated against ground truth, then there is no advantage to testing against the other software rather than against ground truth directly. The primary value in software comparisons is to expose biases produced by different implementations of an algorithm 
when they are run on the same data, or to explore the effects of different settings such as pixel size:feature size or image pre-filtering. The possibilities for these comparisons are many and domain specific, and as such are outside the scope of this manuscript.

Algorithms in BoneJ are validated internally by unit tests on synthetic data for which expected results are readily calculated, and by integration tests that ensure that results on real test images are stable.

Competing Interests: No competing interests were disclosed. 